\title{
Near-Field Microwave Imaging using a Polarimetric Array of 3D Printed Antennas and Lenses
}

\author{
Sebastian G. Wirth*, Ivor L. Morrow* and Ian Horsfall* \\ ${ }^{*}$ Cranfield University, Laboratory of Electromagnetic Systems Engineering, \\ Shrivenham Campus, Oxfordshire, SN6 8LA, UK. \\ Email:Sebastian.Wirth@cranfield.ac.uk,I.L.Morrow@cranfield.ac.uk
}

\begin{abstract}
A novel near-field microwave imaging system was designed and fabricated using the three-dimensional (3D) printing technique to manufacture X-band Pyramidal horn antennas and planar graded-index (GRIN) lenses. The flat lens focusing profile is synthesised by varying the refractive index radially in incremental steps that adjust the air-dielectric mixture. The lens is designed for direct attachment to the antenna aperture and transforms spherical waves emanating from antenna phase center into plane radiating waves. Simulated and measurement results show the antenna lens system input impedance is $\leq-10 \mathrm{~dB}$, radiation pattern gain is between $17-20 \mathrm{dBi}$ over the $8.2-12.4 \mathrm{GHz}$ frequency band and when arrayed for polarimetry sensing has a polarisation cross-talk of $\leq-50 \mathrm{~dB}$. A ground penetrating radar system using the nearfield array was scanned over buried targets. The SAR results demonstrated high resolution and polarisation discrimination imagery capable of detecting subsurface objects.
\end{abstract}

\section{Introduction}

There is an ever increasing interest for next generation ground penetrating radar (GPR) antennas to be lighter and cheaper while performing at least as well as, or better than, conventional antenna types. Moreover, GPR antennas for humanitarian demining applications are required to operate in the near or quasi-near field of the terrain being surveyed. This paper describes the use of additive 3D print manufacture to design practical microwave antennas and lens components which exploit the low cost and light weight advantages of the technique and permit bespoke design features that would otherwise be difficult or expensive to procure commercially. Moreover, the lens enables transmission and reception of linear plane polarised waves in near-field GPR operation and provides a focusing action in addition to any synthetic aperture radar (SAR) processing applied.

The 3D printing of microwave components [1] and lens technology [2] has recently been the subject of much research interest. Usually the lens is located some distance in front of the antenna at the near-field/far-field boundary. A variety of design approaches and 3D printing techniques have been recently reported. Hyperbolic profile lenses have been transformed to equivalent flat lens designs. The refractive index may be made to vary by fabricating the lenses as a series of sub-wavelength unit cell with tailored dielectric value. The range of dielectric values obtained by this $3 \mathrm{D}$ printing technique can introduce constraints on lens thickness. Recent efforts [3], [4] have investigated 3D printing metal particles and dielectrics. These artificial dielectrics are reported to have larger dielectric value permitting construction of thinner lenses. Alternatively, metamaterial surfaces based on slots cut in cascaded dielectrics have been proposed [5] at millimeter wave frequencies. Here the authors consider insertion of a constant thickness lens into the antenna aperture. This rapidly converts near-field spherical wavefronts emerging from the antenna phase center into planar phase fronts with enhanced directivity in the desired direction [6].

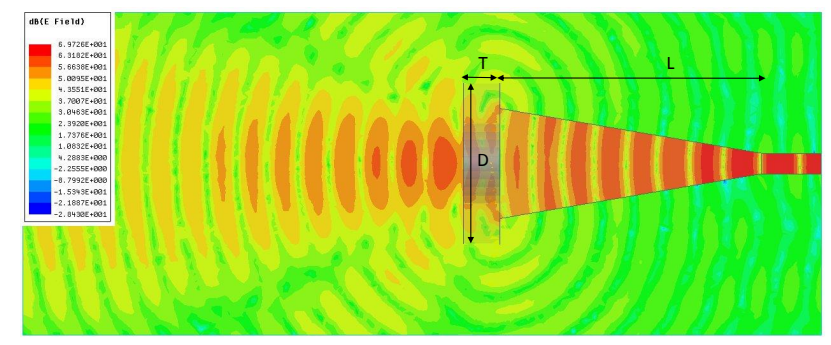

Figure 1. Simulated electric field of the near-field GRIN lens at $10 \mathrm{GHz}$, fed by a rectangular X-band pyramidal horn. The lens is attached to the horn aperture and focused at the antenna phase center: $\mathrm{D}=80 \mathrm{~mm}, \mathrm{~T}=10 \mathrm{~mm}$, $\mathrm{L}=142 \mathrm{~mm}$, WR90 $(10.16 \times 22.86) \mathrm{mm}$.

This paper is organised as follows; Section 2. describes the novel near-field lens design and 3D printing technique. Fig 1 illustrates a full-wave simulation of the electric field emerging from the lens integrated with an X-band Pyramidal horn antenna computed using the commercial software HFSS [7]. The horn antenna is also 3D printed and electroplated with a 40 micon thick copper coating. The horn, although not the subject of this paper, was designed according to standard pyramidal horn equations [8] to provide an optimum gain of $16.3 \mathrm{~dB}$ at $10 \mathrm{GHz}$.

HFSS simulation results for radiation patterns at 8,10 , and $12 \mathrm{GHz}$ frequencies determined the antenna phase center was located $50 \pm 1.0 \mathrm{~mm}$ inside the antenna aperture. The radiation pattern amplitude and phase were measured in an anechoic chamber and results confirmed that experimentally the phase 
center was located between $49-51 \mathrm{~mm}$ behind the horn aperture.

Section 3., shows some of the measured and simulated results for the horn antenna with the lens attached. In Section 4., four near-field horn antennas are assembled into a polarimetry array and polarimetry cross-talk levels characterised. The full polarimetry microwave array is attached to a GPR and scanned over a ground scene where various objects are buried. The polarimetry SAR results are critically evaluated and some conclusions drawn in Section 5.

\section{Flat Lens Design}

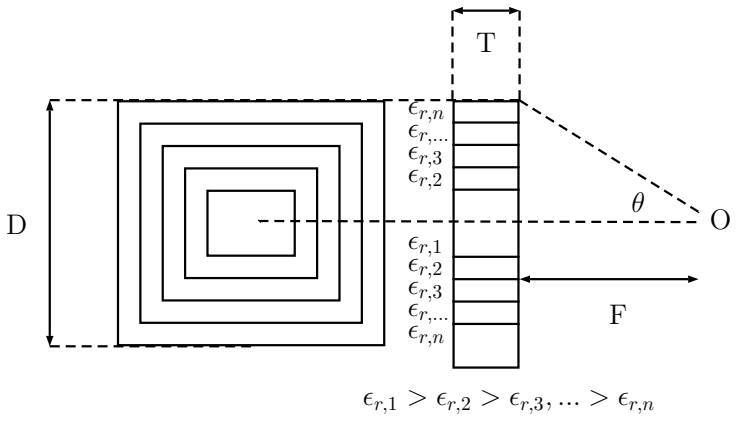

(a) front view

(b) side view

Figure 2. Sketch showing the constant thickness lens composed of annular rectangular ring of decreasing dielectric constant material.

Fig. 2 shows a constant thickness, $T$, rectangular shaped dielectric lens ${ }^{2}$ composed of variable refractive index medium $\left(n=\sqrt{\varepsilon_{r}}\right)$ and with focal point $F$ located on its axis of symmetry. The refractive index decreases linearly from a maximum at the centre of the lens to a value of 1.0 at the perimeter. One feasible 3D printing technique that would approximate this variation is incremental stepping of the refractive index. This permits fabrication as a series of annular rectangular sections of increasing size and decreasing permittivity towards the lens perimeter. The relationship between the radially varying $\varepsilon_{r}$ and angle of incidence $\theta$ can be shown to be [9],

$$
\begin{array}{r}
T\left(\varepsilon_{r}-\frac{2}{3} \sin ^{2}(\theta)\right)=\sqrt{\varepsilon_{r}-\sin ^{2}(\theta)} \times \\
{\left[\sqrt{\varepsilon_{m}} T-(\sec (\theta)-1)\right] .}
\end{array}
$$

where $T$ is the lens thickness, $\varepsilon_{m}$ the maximum permittivity of the lens at the centre and $\varepsilon_{r}$ is the relative permittivity of a particular annular section at $\theta$ degrees to the focal point. Eqn. 1 is the basic design equation for the planar graded refractive index (GRIN) lens and is solved by the root bisection method in Table 1 to determine discrete values of $T$ for $\theta$. An initial estimation of the lens proportions may be made knowing the maximum value of $\theta=55^{\circ}$ is determined by the diameter of the lens and the focal length is fixed at $F=50 \mathrm{~mm}$. A

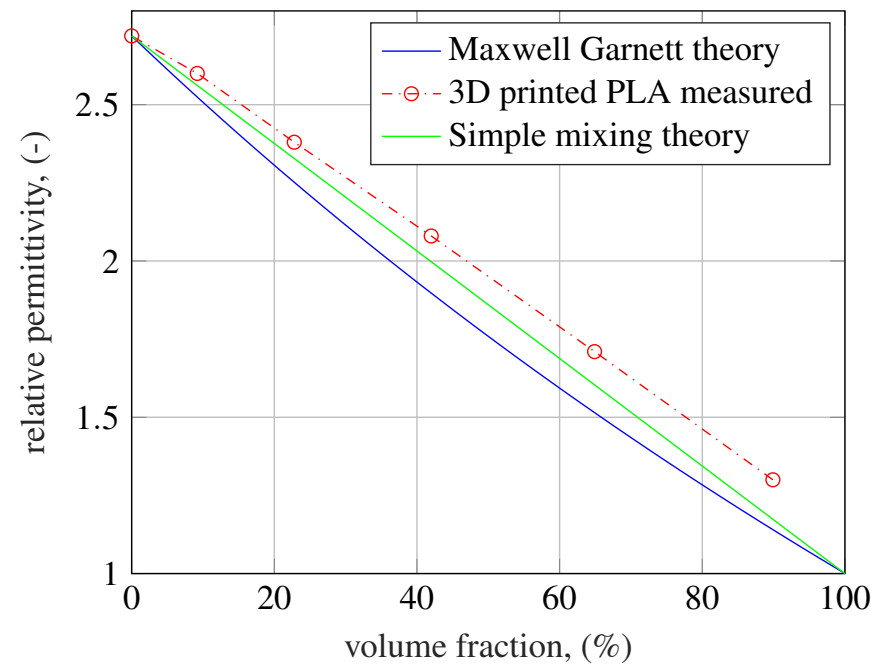

Figure 3. Comparison of effective permittivity model prediction and measured permittivity for PLA and air over a range of volume fractions.

fabrication constraint is also imposed on the minimum value of $\varepsilon_{r}$ which can be printed and maintain the lenses structural strength. A feasible approach is to synthesis "artificial" dielectric values between 2.7- 1.3. One practical method is to exploit dielectric mixing principles and introduce shaped air voids into the base material. The Maxwell-Garnett, with Rayleigh approximation may be solved to determine the effective dielectric constant of the mixture [10] as:

$$
\varepsilon_{e f f}=\varepsilon_{m} \frac{2 \delta_{i}\left(\varepsilon_{i}-\varepsilon_{m}\right)+\varepsilon_{i}+2 \varepsilon_{m}}{2 \varepsilon_{m}+\varepsilon_{i}+\delta_{i}\left(\varepsilon_{m}-\varepsilon_{i}\right)}
$$

The 3D printer base medium, is Poly Lactic Acid or PLA with permittivity $\varepsilon_{m}=2.72$ and the air inclusion permittivity $\varepsilon_{i}=1.0004$, where $\delta_{i}$ is the volume fraction of the inclusion. Fig 3 illustrates the relationship for effective permittivity for air inclusion volume fractions using; (i) Equation (2), (ii) measurement of some 3D printed PLA samples (published in [2]) and (iii) a simple linear volume mixing relationship. Clearly, the effective permittivity follows a nearly linear relationship. Equation (1) was solved subject to the constraints imposed on practical realisation of effective dielectric value and a fixed focus length. The results are summarised in Table 1. The ideal lens thickness is between $47-51 \mathrm{~mm}$ and a trade-off value of $50 \mathrm{~mm}$ thickness was chosen.

The lens is comprised of seven concentric rectangular sections, each with a annular width of $10 \mathrm{~mm}$. Two approaches were taken to 3D printing the dielectric lens; the first was to 3D print the lens as a single whole piece and the second involved 3D printing each annulus separately and carefully push fitting together to form one whole lens. The latter approach was adopted since it allowed flexibility in modifying individual rings without reprinting the whole structure. The HFSS model of the solid lens was translated to the SolidWorks file format. The CAD file was then imported to Slic3r as an .stl file and densities assigned according to Table 1 . The annular sections were $3 \mathrm{D}$ printed using an Ultimaker 3 Extended printer with 
Table 1. Summary design data on the GRIN lens. Tabulated values of lens thickness versus annular rectangular section radius, effective dielectric constant and PLA/air volume fractions for the seven annular sections (the * value indicates a suspect $T$ value).

\begin{tabular}{llllll} 
Ring No. & $\mathrm{R}(\mathrm{mm})$ & $\theta(\mathrm{degs})$ & $\mathrm{T}(\mathrm{mm})$ & $\varepsilon_{\text {eff }}$ & $\delta_{i} \%$ \\
\hline 1 & 10 & 5.0 & 49.69 & 2.72 & 100 \\
2 & 20 & 15.0 & 48.49 & 2.60 & 90.8 \\
3 & 30 & 25.0 & 45.96 & 2.38 & 77.1 \\
4 & 40 & 35.0 & 45.02 & 2.08 & 58.0 \\
5 & 50 & 45.0 & $43.70^{*}$ & 1.71 & 35.1 \\
6 & 60 & 55.0 & 47.85 & 1.30 & 10.1 \\
7 & 70 & 65.0 & 47.31 & 1.20 & 9.89 \\
\hline
\end{tabular}

a $0.4 \mathrm{~mm}$ extrusion nozzle. The completed 3D printed GRIN lens is shown in Fig 4.

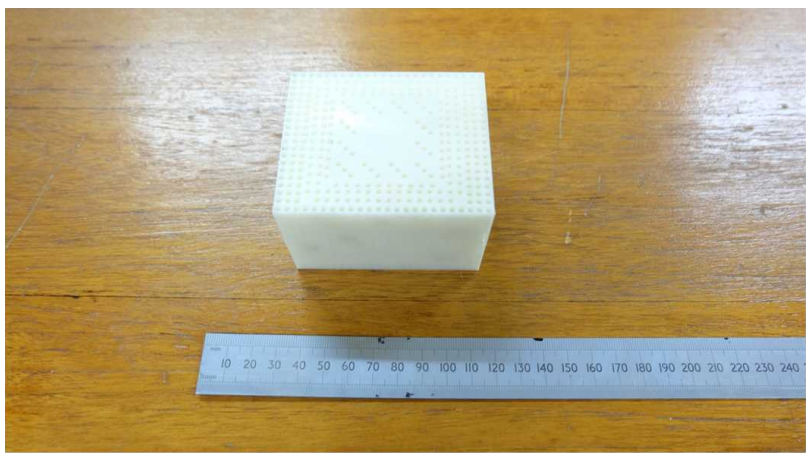

Figure 4. Photograph of the rectangular printed flat GRIN lens.

\section{Results of Antenna and Lens Integration}

Simulation models for the X-band Pyramidal horn and GRIN lens were developed using HFSS. The lens was modeled as seven solid rectangular annular sections of homogeneous dielectric constant value according to Table 1 and assuming negligible loss tangent $\tan \delta=0.008$. Fig. 5 shows the reflection coefficient for the $3 \mathrm{D}$ printed horn antenna with and without the lens attached to the antenna aperture. Agreement between simulation and measurement is good. It is observed that the near-field lens has not affected the impedance match and appears to have improved it at higher frequencies. This implies that the introduction of any impedance matching layer is not required. Fig 6 shows the measured and simulated gain are also in good agreement, the near-field lens has increased the antenna gain by $2.4-3.0 \mathrm{~dB}$ across the $8.2-12.4 \mathrm{GHz}$ bandwidth and introduced a perturbation in the gain.

\section{Polarimetric Near-field SAR and First Results}

An X-band polarimetry SAR system has been developed as part of the dual (UHF- to S-band) frequency, polarimetry GPR system. The transmitter power is $0 \mathrm{dBm}$. The antenna array shown in Figure 7 implements two pairs of horizontally and vertically polarised antennas to achieve polarisation diversity and the figure inset depicts the horn antenna aperture orientation with the lenses removed. The GPR system is configured

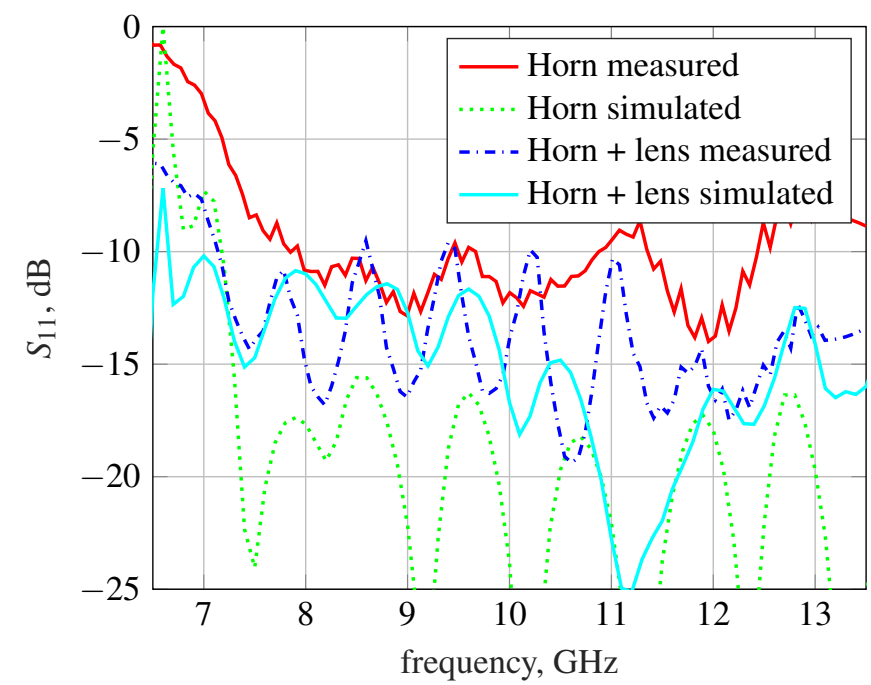

Figure 5. Measured and simulated reflection coefficient of 3D printed horn antenna with and without near-field lens.

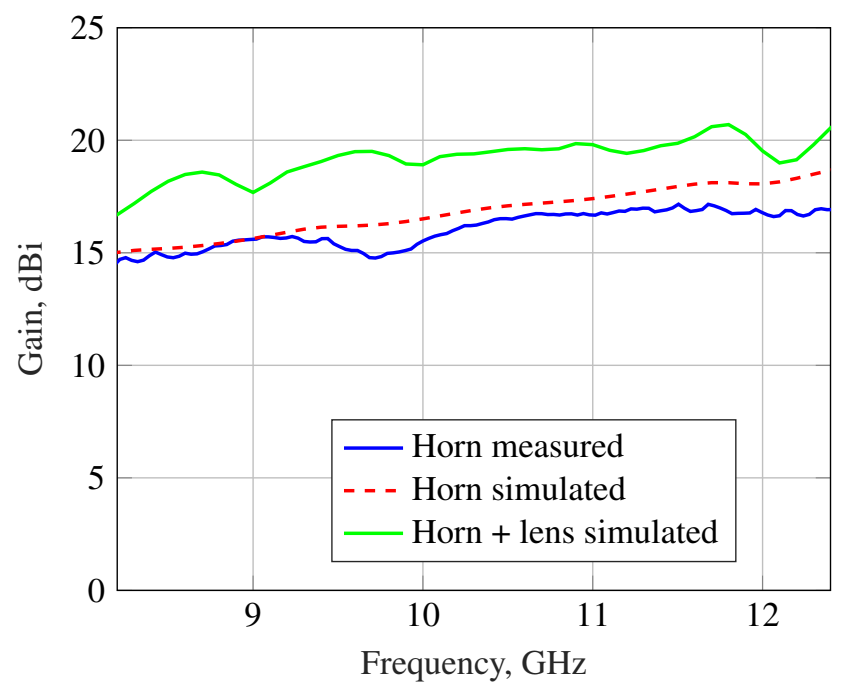

Figure 6. Measured and simulated gain of 3D printed horn antenna with and without near-field lens.

to sequentially measure the scattering parameter $S_{21}$ across the X-band $(8.2-12.4 \mathrm{GHz})$ frequency spectrum in all possible combinations available from the $\mathrm{H}$ - and $\mathrm{V}$-antennas, i.e. $\mathrm{H}$ transmitting and H-receiving and soforth). Then the full scattering scattering matrix $(\mathrm{HH}, \mathrm{HV}, \mathrm{VH}, \mathrm{HH})$ data for a given position can be collected. The antenna array is horizontally scanned from a height of $50 \mathrm{cms}$ above the ground surface. The GPR provides a depth of penetration of $60 \mathrm{cms}$ in soils with moisture levels below $3 \% \mathrm{w} / \mathrm{w}$. The array consists of four standard gain horn antennas, two are 3D printed and the other two are Narda Mod. 640 horn antennas. This setup permits some comparisons of $3 \mathrm{D}$ printed antenna performance with that of the commerical horn antenna pair where co-polar channel scattered responses are equal for e.g. from a sphere. The lenses are then attached using a thin layer of cyanoacrylate compound. The polarisation switch is a conventional PIN diode switch 
matrix able to switch $30 \mathrm{dBm}$ peak power from the transmitter while exhibiting low loss $(0.3 \mathrm{~dB})$ and high isolation (more than $50 \mathrm{~dB}$ ). Thus system cross-talk (between polarisations) is dominated by antenna cross-talk. Fig. 8 demonstrates the measured $S_{11}$ response for all the antennas in the polarimetry array with lenses attached. All the antennas are well matched over the $8.2-12.4 \mathrm{GHz}$ operating frequency. Figs. 9 and 10 show the measured co-polar mutual coupling in E- and H-planes of the polarimetry array are less than $-50 \mathrm{~dB}$ and the cross-polar mutual coupling in E- and H-planes is less than $-60 \mathrm{~dB}$.

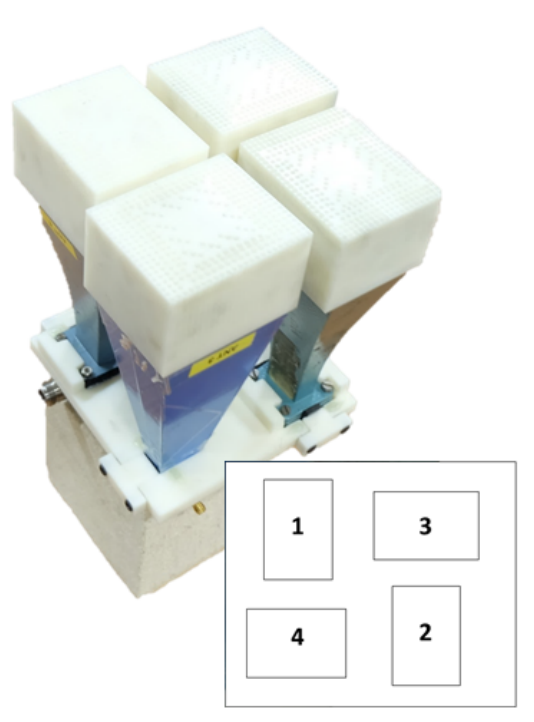

Figure 7. Polarimetry array incorporating two 3D printed horn antennas and two commercial horns; lenses are shown attached for clarity the inset depicts the orientation of the horn apertures, when looking directly at the array, the numbers identify individual antennas, 1 and 2 are the 3D printed antenna pair designed as VV polarisation (E-field in plane of incidence and direction of scanner motion) and 3 and 4 are the Narda horn antennas designated as $\mathrm{HH}$ polarisation (E-field orthogonal to the plane of incidence and direction of scanner motion).

Polarimetric imagery was acquired over a laboratory test lane consisting of a sand bay approximately $(3.0 \times 1.2 \times 0.8)$ $\mathrm{m}$ in square area and depth and shown in Fig. 11. Located in the scene is a buried anti-personnel landmine as well as several calibration targets. These include a metal sphere, a dihedral rotated $22.5^{\circ}$ to its horizontal seam to provide equal co- and cross-polar returns and buried trihedral to provide a strong co-polar response. The calibrated polarimetry SAR scan imaging results for the antenna array without lenses are shown in Fig 12. It is evident that all the objects are present in $\mathrm{VV}$ and $\mathrm{HH}$ polarisations. The dihedral, as would be anticipated, is also present with an equally strong signal power in cross-polarisations, $\mathrm{HV}$ and $\mathrm{VH}$. The sphere and trihedral have no cross-polar return as would be expected. The PMN-1 landmine back scatter response is a little stronger in VV than $\mathrm{HH}$ polarisation while absent in both $\mathrm{HV}$ and $\mathrm{VH}$ polarisation. This experiment demonstrates how polarimetry can be used to effectively discriminate buried objects and this is well known. The calibrated polarimetry SAR scan imaging results for the

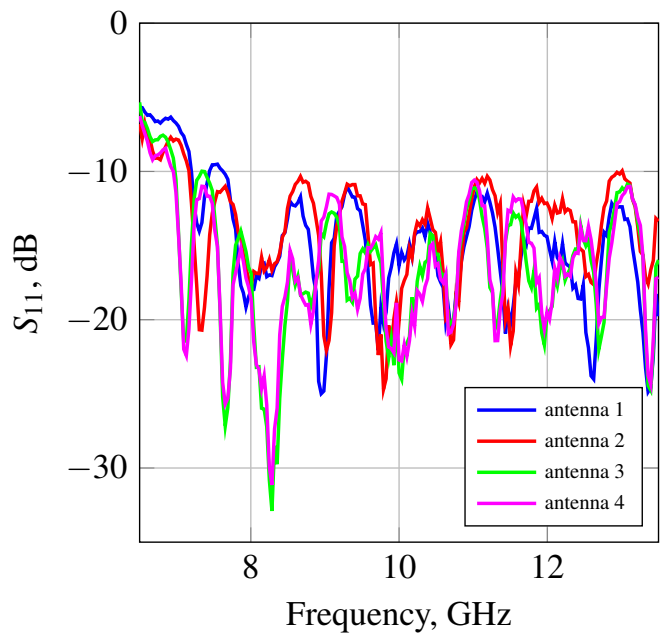

Figure 8. Measured $S_{11}$ reflection coefficient for individual antennas in the polarimetry array (see Fig. 7 inset to locate individual antennas).

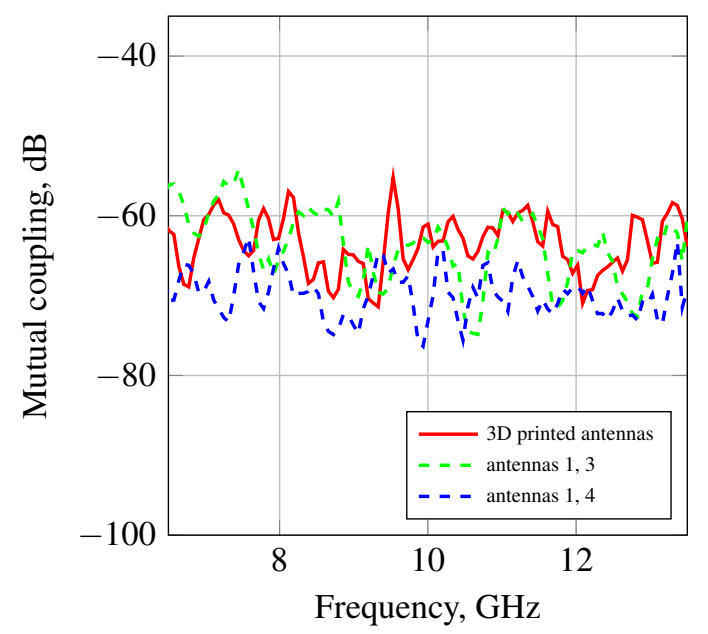

Figure 9. Co- and cross-polar mutual antenna coupling in E-plane (see Fig. 7 inset to locate individual antennas).

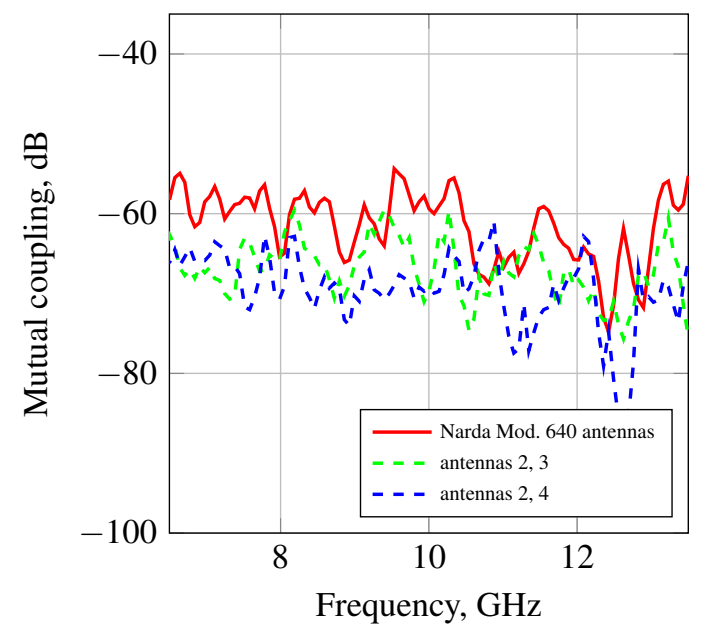

Figure 10. Co- and cross-polar mutual antenna coupling in H-plane (see Fig. 7 inset to locate individual antennas). 
antenna array with lenses attached are shown in Fig 13. All calibration objects and buried targets are again present and shifted down range a few centimeters, however they are more localised than those processed without the lenses attached which is due to the enhanced gain of the lensed array.

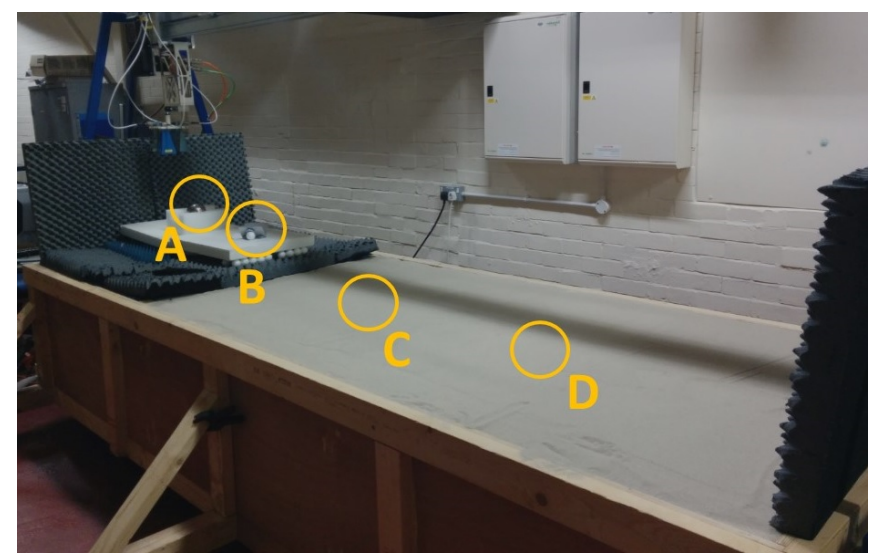

Figure 11. Laboratory sand bay with several calibration objects and a PMN-1 landmine located at the positions shown; A: $60 \mathrm{~mm}$ metal sphere, B: $100 \mathrm{~mm}$ square sided dihedral, C: $70 \mathrm{~mm}$ sided trihedral buried $90 \mathrm{~mm}$ in depth and D: $100 \mathrm{~mm}$ dia. $\mathrm{x} 40 \mathrm{~mm}$ thick PMN-1 anti-personnel landmine buried $100 \mathrm{~mm}$ in depth.

\section{Conclusions}

This paper has described the 3D design and fabrication of lowcost, light-weight planar microwave lens tailored for near-field sensor applications. The lens is attached to an X-band horn antenna aperture and provides an enhanced gain of 2.4-3.0 dB over that of the horn antenna without a lens. The lens face is $80 \times 70 \mathrm{~mm}$ in area and $50 \mathrm{~mm}$ thick with an effective permittivity that varies radially from a maximum at the center to a minimum at the edges. The antenna input impedance and gain performance with the lens attached demonstrated good correlation between simulation and measurements. Four antennas with lenses attached were arranged in a polarimetry array and measurements indicated the antenna cross-talk level below 50dB. The near-field sensor was deployed in a GPR application and polarimetry scans of some calibration objects and buried targets demonstrated the additional antenna gain combined with SAR focusing provided enhanced spatial resolution and contrast SAR imagery.

\section{Acknowledgements}

The authors thank the Find A Better Way charity for their support of this research under the DETERMINE programme (grant number 2015/001D).

\section{References}

[1] K. V. Hoel and S. Kristoffersen and J. Moen and K. G. Kjelgrd and T. S. Lande, Broadband antenna design using different $3 \mathrm{D}$ printing technologies and metallization processes, 10th European Conference on Antennas and Propagation (EuCAP), pp. 1$5,(2016)$
[2] S. Zhang and R. K. Arya and S. Pandey and Y. Vardaxoglou and W. Whittow and R. Mittra, 3D-printed planar graded index lenses, IET Microwaves, Antennas Propagation, vol. 10, no. 13, pp. 1411-1419, (2016).

[3] S. Zhang and W. Whittow and J. C. Vardaxoglou, Additively manufactured artificial materials with metallic meta-atoms, IET Microwaves, Antennas Propagation, vol. 11, no. 14, pp. 19551961, (2017).

[4] P. Petrov and A. Radkovskaya and C. J. Stevens and E. Shamonina, Superdirectivity for coupled dimers of meta-atoms at $\mathrm{MHz}$, 11th International Congress on Engineered Materials Platforms for Novel Wave Phenomena Metamaterials, pp. 265-267, (2017).

[5] M Al-Nuaimi, Compact Size High Gain Lens Corrected Pyramidal Horn Antenna for 71-76 GHz Band, Third Asia Pacific Conference on Antennas and Propagation, pp. 23-26, (2014).

[6] T. Ding and J. Yi and H. Li, 3D field-shaping lens using alldielectric gradient refractive index materials, Scientific Reports 7.782, DOI:10.1038/s41598-017-00681-z, (2017).

[7] Ansys HFSSv18.1, Getting Started with HFSS: Begineers Guide, http: //www . ansoft . com, (2016).

[8] C.A. Balanis, Antenna Theory, Wiley, ISBN:0-471-66782-X, (2005).

[9] H. Jasik, Antenna Engineering Handbook, MCGraw-Hill Book Company, Chap.16, (1961).

[10] A. Sihvola and J. Kong, Effective Permittivity of Dielectric Mixtures, IEEE Trans. on Geoscience and Remote Sensing, vol. 26, no. 4, pp. 420-429, (2000). 
$\mathrm{HH}$

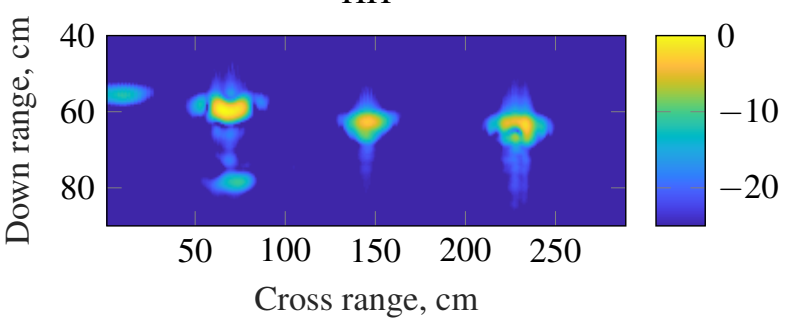

$\mathrm{VH}$

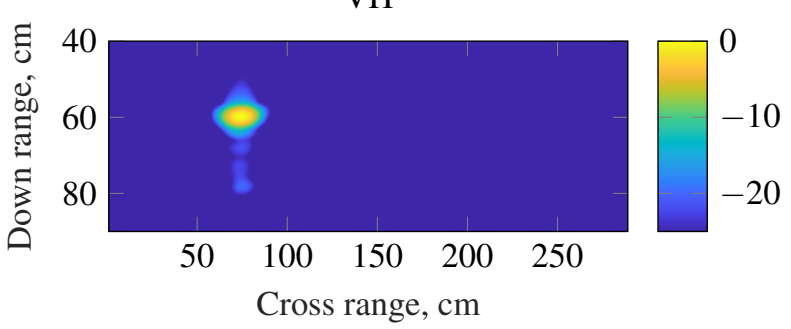

HV

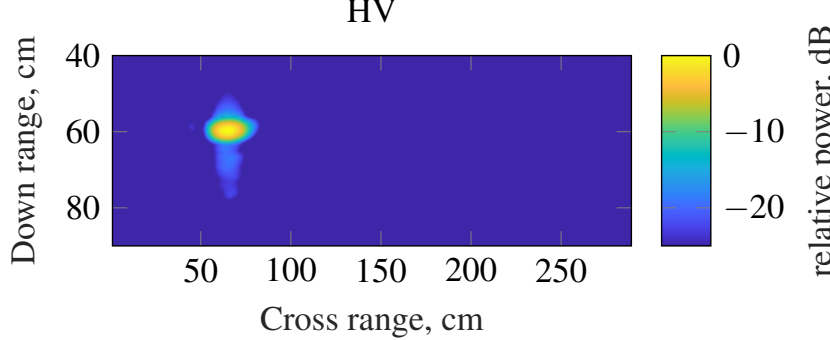

VV

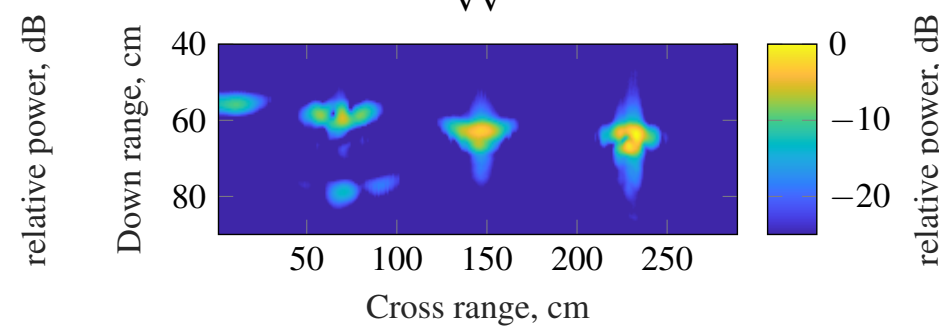

Figure 12. Polarimetric SAR scans without lensed array of the sand bay scene. Various calibration objects and buried targets in the ground as indicated in Fig. 11 (GPR parameters: frequency sweep $8.2-12.4 \mathrm{GHz}$, waveform type SFCW, No. of frequency steps = 801, measurement steps $\Delta x=1.0 \mathrm{~cm}$; GPR processing: background subtracted, windowed using 4-term Blackman Harris and synthetic aperture processed).
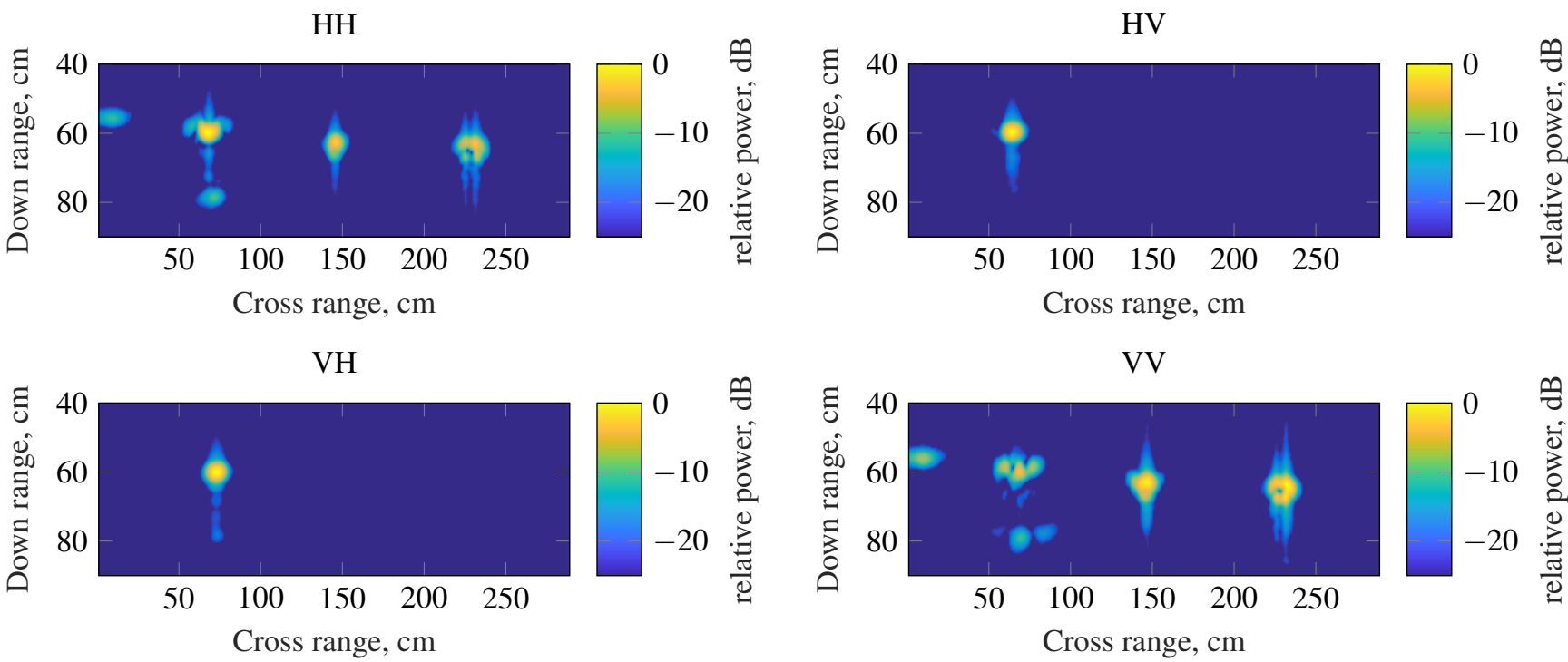

Figure 13. Polarimetric SAR scans with lensed array of the sand bay scene. Various calibration objects and buried targets in the ground as indicated in Fig. 11 (GPR parameters: frequency sweep $8.2-12.4 \mathrm{GHz}$, waveform type SFCW, No. of frequency steps $=801$, measurement step $\Delta x=1.0 \mathrm{~cm}$; GPR processing: background subtracted, windowed using 4-term Blackman Harris and synthetic aperture processed). 